\title{
Histomorphometric changes following treatment for osteoporosis
}

\author{
C. A. Moreira $^{1}$ D. W. Dempster ${ }^{2,3}$
}

Received: 23 February 2017 / Accepted: 22 March 2017 / Published online: 26 May 2017

(C) Italian Society of Endocrinology (SIE) 2017

A significant body of information is available regarding the effects of osteoporosis drugs on bone histomorphometry $[1,2]$. This is, in part, because regulatory agencies require biopsies to be performed to assess the safety and mechanism of action of new therapeutic agents. There are two classes of osteoporosis drugs: antiresorptive and anabolic. As revealed by bone histomorphometry, each class has a fundamentally different mechanism of action. Antiresorptive therapies, such as estrogen, calcitonin, raloxifene, bisphosphonates, and denosumab, decrease bone turnover, lowering both resorption, and formation [2]. There are several histomorphometric studies on the effect of hormone therapy (HT) in postmenopausal women, providing convincing evidence of its beneficial skeletal action in preserving bone volume and structure by decreasing bone turnover. The reduction in bone remodeling parameters after treatment with HT is more pronounced than that observed with raloxifene or calcitonin. Similarly, bone biopsy studies following treatment with bisphosphonates show a reduction in the rate of bone remodeling with a decrease in osteoid surface and in the tetracycline-based, dynamic parameters of bone turnover: mineralizing surface, bone formation rate and activation frequency. However, a study analyzing biopsies obtained after 2-3 years of alendronate treatment

D. W. Dempster

ddempster9@aol.com

1 Division of Federal University of Parana (SEMPR), Department of Internal Medicine and Bone Unit of Endocrinology, Laboratory P.R.O. at Pro Renal Foundation, Curitiba, PR, Brazil

2 Regional Bone Center, Helen Hayes Hospital, West Haverstraw, New York, USA

3 Department of Pathology, College of Physicians and Surgeons of Columbia University, New York, NY, USA demonstrated that mineral apposition rate (MAR) was unchanged, confirming that there is no inhibition of bone mineralization, despite the reduction in bone remodeling rate. Furthermore, alendronate had minimal effect on the parameters related to bone resorption such as osteoclast number and eroded surface. Bone microarchitecture, cancellous bone volume, and cortical thickness are preserved with antiresorptive treatments. After 3 years of treatment with risedronate, a paired biopsy study in postmenopausal women showed a decrease in bone remodeling associated with preservation of bone structure. The diminution of bone remodeling with risedronate is less than that which occurs with zoledronic acid and denosumab, which are considered to be more potent antiresorptive agents.

In the FREEDOM trial of denosumab bone, biopsies were performed after 24 or 36 months of treatment [3]. They showed a significant reduction in eroded surface and osteoclast number with denosumab compared to placebo. In addition, dynamic parameters were reduced by denosumab treatment with the median bone formation rate being reduced by $97 \%$ in comparison to placebo. In fact, double tetracycline labeling in trabecular bone was observed in only $40 \%$ of the patients treated with denosumab, while it was present in $100 \%$ of the placebo group. However, it is important to note that, among patients on denosumab, those who had tetracycline labels and those without labels had similar circulating bone turnover marker levels. This finding suggests that lack of labels at the iliac crest does not necessarily mean absence of bone remodeling in other skeletal sites [3]. The STAND trial compared denosumab to alendronate and showed that indices of bone turnover were lower in patients treated with denosumab than with alendronate, confirming the more potent antiresorptive effect of denosumab seen with biochemical markers [3]. Furthermore, it is important to note that the effects of denosumab 
at tissue level are fully reversible after its discontinuation and this has $\mathrm{s}$ been demonstrated by histomorphometric studies.

Anabolic therapies work by a fundamentally different mechanism of action than antiresorptive agents [2, 4] (Fig. 1). Rather than reducing the activation frequency of bone remodeling, they increase it and the amount of new bone formed, in each remodeling transaction exceeds the amount that was removed, resulting in an increase in bone mass. Rather than simply maintaining bone microarchitecture, anabolic therapies improve it. To date most of the published studies have evaluated bone histomorphometry following treatment with teriparatide. Paired biopsies in postmenopausal women clearly demonstrated its anabolic effect on bone revealed by improved trabecular and cortical bone microarchitecture associated with increases in osteoblast, osteoid, and mineralizing surface, bone formation rate, as well as in the thickness of bone packets. Teriparatide is therefore characterized as a pro-remodeling anabolic agent. While the increased bone formation rate is largely the result of increased remodeling, teriparatide treatment also increases modeling-based bone formation, i.e., bone formation without prior resorption. The increase in both types of bone formation provides the mechanism for the increases in bone volume, trabecular connectivity, and cortical thickness, confirmed by 3D-micro-CT analysis. Stimulation of bone formation by teriparatide has recently been demonstrated in the femoral neck of patients undergoing total hip replacement [5]. This is the first study to examine the effect of any osteoporosis medication on the human proximal femur, the site of most serious of osteoporosis-related fractures.

The effects of strontium ranelate (SrRan) on bone histomorphometry are modest. A recent large, paired biopsy study [6] compared the effects of SrRan with those of alendronate over 1 year. Bone remodeling parameters were higher with SrRan than with alendronate, but there was no evidence of osteoanabolic action.

Recently, the effects of a new anabolic agent have been studied at the tissue level. Abaloparatide-SC is a potent and selective activator of the parathyroid hormone receptor (PTH1R) signaling pathway which binds to PTH1R with a different affinity for the $\mathrm{R}^{0}$ configuration [7]. Qualitative histological analysis of biopsies from postmenopausal women taking abaloparatide-SC revealed normal bone microarchitecture and bone quality. Like teriparatide, abaloparatide-SC is a pro-remodeling anabolic agent. In a comparison of biopsies from patients taking placebo, abaloparatide-SC, or teriparatide for up to 18 months, there were few differences in the histomorphometric indices
Fig. 1 Tetracycline labels in the cancellous bone of iliac biopsies taken from subjects treated with teriparatide or zoledronic acid for 6 or 24 months. Note extensive labeling in the teriparatidetreated subjects compared with few labels in the zoledronic acid-treated subjects, consistent with the contrasting effects of the two agents on the bone turnover rate. In the lower right image, the labels administered at 6 and 24 months can both be seen, allowing visualization of new bone laid down under the influence of teriparatide in the intervening 18 months. Reproduced with permission from [4]

\section{Zoledronic Acid}

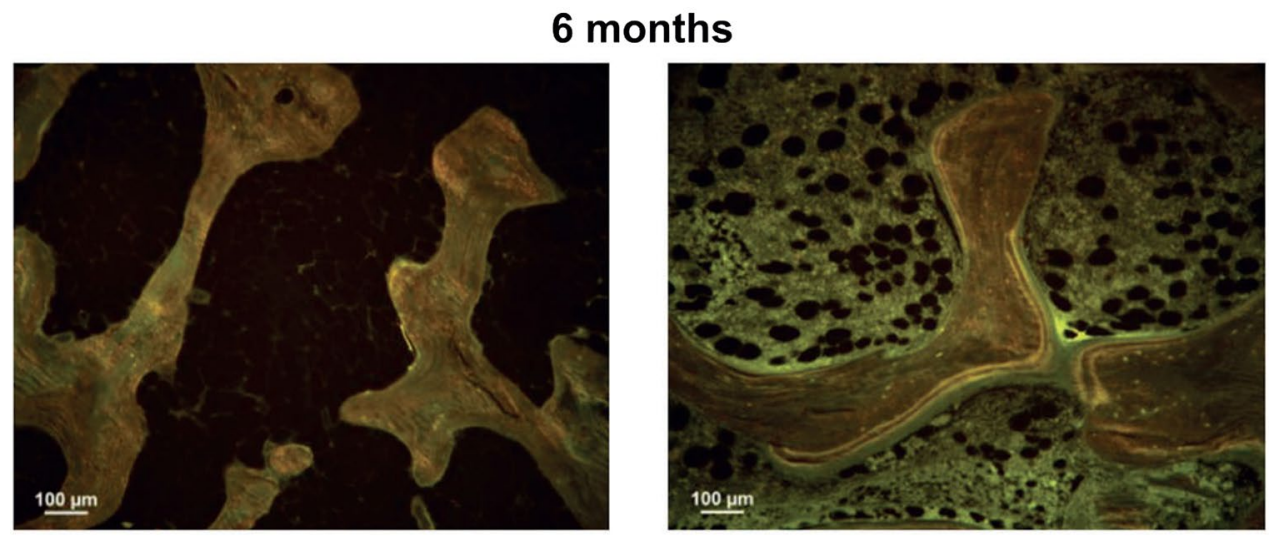

24 months

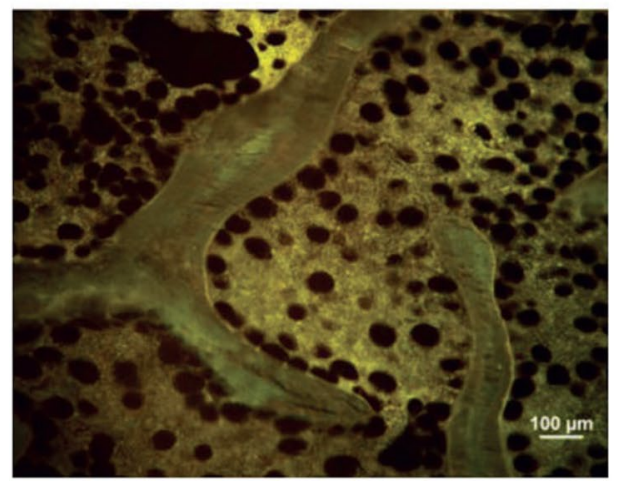


between the two agents; eroded surface was lower in the abaloparatide-SC-treated group, and MAR was higher in the teriparatide-treated group than in the placebo-treated group. Cortical porosity was higher in both the abaloparatide-SC and teriparatide groups than in placebo.

Few studies have examined the effects of combined antiresorptive and anabolic treatment at the tissue level. Recently, Dempster et al. [8] showed that teriparatide was able to stimulate bone formation without an increase in cortical porosity in the presence of prior and ongoing alendronate treatment.

In conclusion, trans-iliac bone biopsy and histomorphometry has played, and will continue to play an invaluable role in the evaluation of the safety and mechanism of action of osteoporosis medications.

\section{Compliance with ethical standards}

Conflict of interest Dr. Moreira serves as a consultant for Amgen and on the Speakers Bureaus for Eli Lilly \& Co. and Amgen, Inc. She has served as a principal investigator on clinical trials sponsored by Amgen Inc and Radius Pharmaceuticals. Dr. Dempster serves as a consultant for Eli Lilly \& Co, Amgen, Inc., Radius Health, and Tarsa Therapeutics and serves on the Speaker's Bureaus for Eli Lilly \& Co. and Amgen, Inc.

\section{References}

1. Kulak CA, Dempster DW (2010) Bone histomorphometry: a concise review for endocrinologists and clinicians. Arq Bras Endocrinol Metabol 54(2):87-98
2. Zhou H, Dempster DW (2013) Lessons from bone histomorphometry on mechanisms of action of osteoporosis drugs. In: Marcus R, Feldman D, Dempster DW et al (eds) Osteoporosis, 4th edn, pp 1777-1803

3. Reid IR, Miller PD, Brown JP, Kendler DL, Fahrleitner-Pammer A, Valter I, Maasalu K, Bolognese MA, Woodson G, Bone H, Ding B, Wagman RB, San Martin J, Ominsky MS, Dempster DW; Denosumab Phase 3 Bone Histology Study Group (2010) Effects of denosumab on bone histomorphometry: the FREEDOM and STAND studies. J Bone Min Res 25(10):2256-2265

4. Dempster DW, Zhou H, Recker RR, Brown JP, Bolognese MA, Recknor CP, KendlerDL, Lewiecki EM, Hanley DA, Rao SD, Miller PD, Woodson GC 3rd, Lindsay R, Binkley N, Alam J, Ruff VA, Gallagher ER, Taylor KA (2016) A longitudinal study of skeletal histomorphometry at 6 and 24 months across four bone envelopes in postmenopausal women with osteoporosis receiving teriparatide or zoledronic acid in the SHOTZ Trial. J Bone Min Res 31(7):1429-1439

5. Cosman F, Dempster DW, Nieves JW, Zhou H, Zion M, Roimisher C, Houle Y, Lindsay R, Bostrom M (2016) Effect of teriparatide on bone formation in the human femoral neck. J Clin Endocrinol Metab 101(4):1498-1505

6. Chavassieux P, Meunier PJ, Roux JP, Portero-Muzy N, Pierre M, Chapurlat R (2014) Bone histomorphometry of transiliac paired bone biopsies after 6 or 12 months of treatment with oral strontium ranelate in 387 osteoporotic women: randomized comparison to alendronate. J Bone Min Res 29(3):618-628

7. Moreira C, Fitzpatrick LA, Wang Y, Recker RR (2016) Effects of Abaloparatide-SC (BA058) on bone histology and histomorphometry: the ACTIVE phase 3 trial. Bone 97:314-319. doi:10.1016/j.bone.2016.11.004 (Epub ahead of print)

8. Dempster DW, Cosman F, Zhou H, Nieves JW, Bostrom M, Lindsay R (2016) Effects of daily or cyclic teriparatide on bone formation in the iliac crest in women on no prior therapy and in women on alendronate. J Bone Min Res 31(8):1518-1526 